\title{
Recording Performances of Perfectly Aligned HSS Heads
}

\author{
Adrian Hozoi and J. C. Lodder
}

\begin{abstract}
The recording characteristics of Helical Scan Silicon (HSS) heads with perfect alignment of the magnetic poles were investigated. Their overall performances were found to be superior to those of ferrite MIG heads, especially the side writing. Erase bands of $0.25 \mu \mathrm{m}$ were measured using a HSS head and ME tape with $50 \mathrm{~nm}$ thick magnetic layer. Side writing and erasing were studied from the frequency response of microtracks, as well as from triple-track profiles.
\end{abstract}

Index Terms-Erase bands, magnetic tape recording, microtracks, side writing, thin-film heads.

\section{INTRODUCTION}

$\mathbf{T}$ HE process of increasing the track density in magnetic tape recording must be supported by a parallel reduction of the erase bands. It was shown in [1] that the erase bands were cut down to $0.4 \mu \mathrm{m}$ in order to reach a track width of $5 \mu \mathrm{m}$ in the MICROMV video system. The improvement came from a better alignment of the magnetic poles of the MIG write head, achieved by mechanical trimming. A recording density of $11.5 \mathrm{~Gb} / \mathrm{in}^{2}$ was reported in [2] using thin ME tape and a GMR read head, without addressing the side writing/erasing issue. However, such density can practically be achieved only if the erase bands are reduced close to $0.1 \mu \mathrm{m}$. Side writing is influenced by a number of factors including the magnetic spacing between head and medium, the medium's thickness and magnetic properties, and the write field gradient at the track edges. The magnetic spacing in tape recording is unlikely to ameliorate much. Tape media with very thin magnetic layers of $20 \mathrm{~nm}$ and high coercivity around $200 \mathrm{kA} / \mathrm{m}$ was obtained by metal sputtering [3], and may be suitable to better control the erase bands. However, most improvements are expected from the write heads that should generate better-confined fields, using well-aligned magnetic poles and short gap length. The Helical Scan Silicon (HSS) heads are an innovative step toward these features [4], [5]. In this work, the recording performances of HSS heads with very good pole alignment were investigated, with great emphasis on their edge effects. Ferrite MIG heads were examined as well. We introduced a technique to study the side writing phenomenon from the frequency response of microtracks. The method allows analyzing the deterioration of the transitions at each track edge as a function of the frequency. Triple-track profiles were also measured to estimate the erase bands of the heads.

Manuscript received October 16, 2003. This work was supported by the Dutch Technology Foundation (STW).

The authors are with the Systems and Materials for Information Storage, MESA+ Research Institute, University of Twente, 7500 AE Enschede, The Netherlands (e-mail: a.hozoi@el.utwente.nl; j.c.lodder@el.utwente.nl).

Digital Object Identifier 10.1109/TMAG.2004.828967

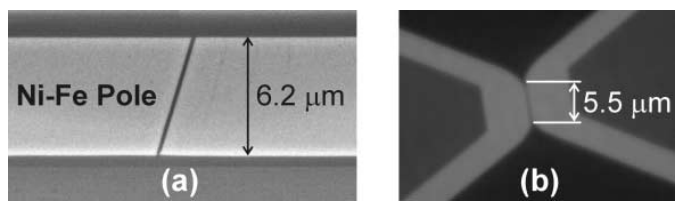

Fig. 1. Magnetic poles of the (a) HSS head and (b) MIG head.

\section{EXPERIMENT}

HSS heads were developed by CEA-LETI and Alditech using advanced thin-film technology [4]. They feature unique properties such as integrated solenoid coil, integrated azimuth angle, and very good alignment of the magnetic poles. Two HSS heads were investigated, having a pole width of $6.2 \mu \mathrm{m}$, electrical gap length of $0.23 \mu \mathrm{m}$, and azimuth angle of $15^{\circ}$. They have solenoid coils with 40 turns, dc resistance below $6 \Omega$, and inductance around $0.6 \mu \mathrm{H}$ (at $20 \mathrm{MHz}$ ). Two MIG ferrite heads with track widths of $5.5 \mu \mathrm{m}$ and $7.0 \mu \mathrm{m}$ were studied as well, and both presented very similar edge effects. Their electrical gap length was the same as that of the HSS heads, i.e., $0.23 \mu \mathrm{m}$. They had an azimuth angle of $10^{\circ}$, a coil with 15 turns and inductance around $0.6 \mu \mathrm{H}$ (at $5 \mathrm{MHz}$ ). Only results obtained with one HSS head and one MIG head (of $5.5 \mu \mathrm{m}$ track width) are presented here, as they are representative of each technology investigated. Images of their magnetic poles are given in Fig. 1.

The recording experiments were performed at $3 \mathrm{~m} / \mathrm{s}$ speed, on a rotating tape-drum tester with cross-track resolution beyond $0.1 \mu \mathrm{m}$. We used ME tape with the magnetic layer's thickness $\delta=50 \mathrm{~nm}$, and the coercivity $H_{c}=135 \mathrm{kA} / \mathrm{m}(1700 \mathrm{Oe})$. This sample was fabricated by Sony Corporation and is similar to the tape used in the MICROMV video system [1].

\section{RESULTS AND DISCUSSION}

The frequency responses of the HSS and MIG heads are given in Fig. 2. The optimum recording current was $10 \mathrm{~mA}$ for the HSS head and $20 \mathrm{~mA}$ for the MIG head. The HSS head needs very low write current, which is unusual for thin-film technology. This is due to its solenoid thin-film coil with high number of turns and good efficiency. The output signal of the HSS head has much higher amplitude and decays less with the frequency, even though the track width is almost the same. The better performance of the HSS head should come from superior sensitivity and weaker edge effects.

The side writing phenomenon was explored by studying the frequency response of microtracks created at the edges of the track as well as in the center. The center-microtracks were prepared by erasing at both sides of the recorded track; the edge- 


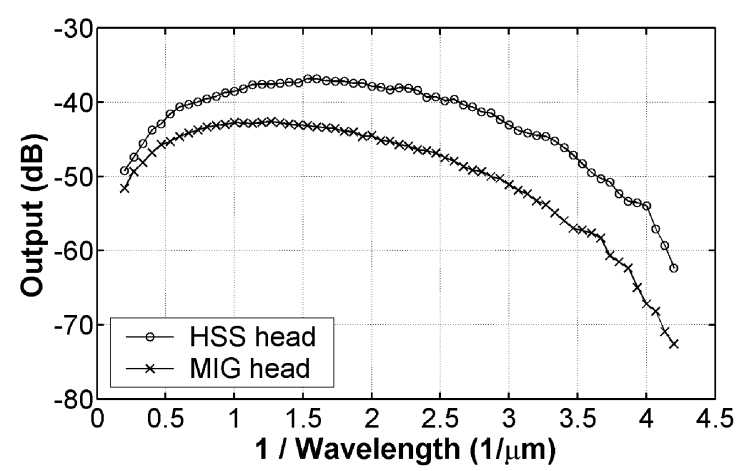

Fig. 2. Frequency response of HSS and MIG heads.

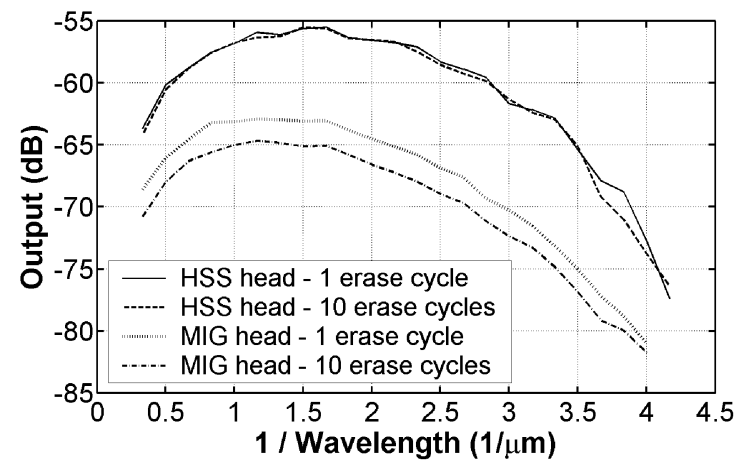

Fig. 3. Influence of the number of erase cycles on microtracks.

microtracks were prepared by erasing at one side only. The erase method used to narrow the recorded track may influence the quality of the microtracks. The erasure has to be complete and leave the edge of the microtrack abrupt and free of distortions. Different erase techniques were studied in order to minimize related artifacts. They were tested by comparing the microtracks prepared using one erase cycle and ten erase cycles. Minimal differences should result with a good erase method. We preferred a method consisting in a cycle of erasing two times with dc currents of opposite sense. The frequency responses of center-microtracks prepared using one and ten erase cycles are shown in Fig. 3, for both HSS and MIG heads. In the case of the HSS head, there is hardly any difference between one and ten erase cycles. This is probably due to a very well confined write field, related to the good pole alignment. The situation is different with the MIG head, where the signal from the microtrack created with ten erase cycles is evidently lower. However, the reduction does not seem to depend much on frequency.

The frequency responses of edge-microtracks obtained with the HSS head are plotted in Fig. 4. After writing a full track, the head was offset with plus or minus $0.8 \mu \mathrm{m}$ to erase part of the track. The erase offset, $E O$, is given in the figure for each curve. The read offset, $R O$, was null when the head was replaced at the original write position for reading. Reading was also performed with the head centered on the microtrack, in which case $R O$ was $+2 \mu \mathrm{m}$ or $-2 \mu \mathrm{m}$. The frequency responses of two centermicrotracks are also included in the same figure for reference. Their erase offsets were $E O=-/+3.45 \mu \mathrm{m}$, and $E O=$ $-/+3.70 \mu \mathrm{m}$.

The edge-microtracks measured with the HSS head exhibit very similar frequency behavior for both edges and read posi-

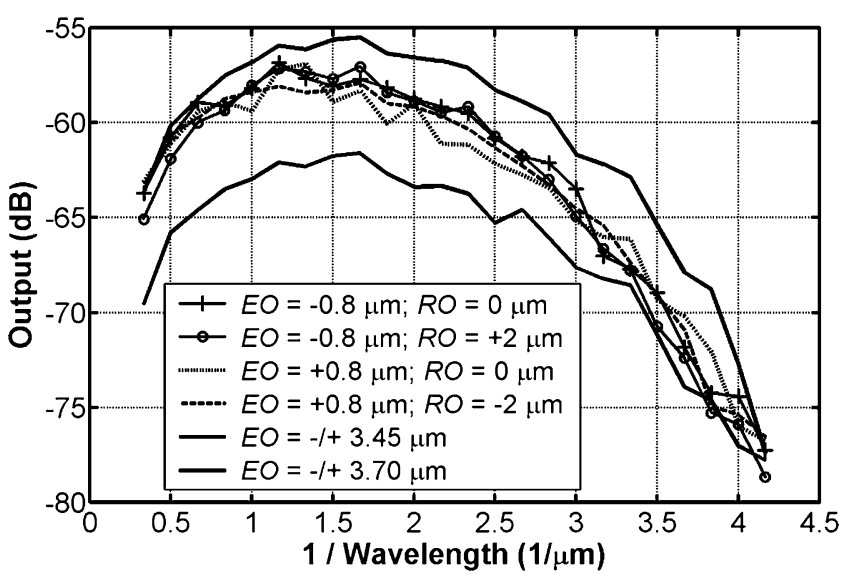

Fig. 4. Frequency response of microtracks measured with the HSS head.

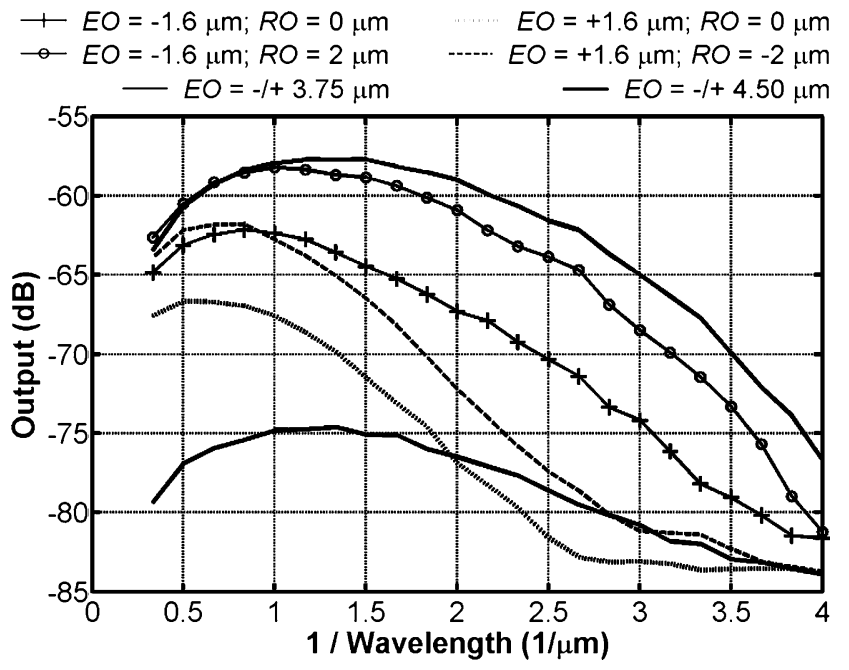

Fig. 5. Frequency response of microtracks measured with the MIG head.

tions. Their frequency decay is higher than of the center-microtracks, due to the degradation of the transitions at the track edges. This can be seen as an effective track width reduction at short wavelengths. The roll-off curves of the edge-microtracks are contained in between the roll-off curves of the two center-microtracks, for the frequency range investigated. The difference between the widths of the center microtracks was deduced from the values of their erase offsets to be $0.5 \mu \mathrm{m}$. We could thus estimate that the effective track width reduction at each edge is less than $0.5 \mu \mathrm{m}$ up to a wavelength of $0.2 \mu \mathrm{m}$. The effective track width reduction at a recording wavelength of $0.33 \mu \mathrm{m}$ was found to be about $0.3 \mu \mathrm{m}$ (from a center microtrack with $E O=-/+3.55 \mu \mathrm{m}$ ).

Edge and center-microtracks measurements were performed with the MIG head as well, and the results are plotted in Fig. 5. The erase offsets had to be increased considerably compared with the HSS head $(1.6 \mu \mathrm{m}$ instead of $0.8 \mu \mathrm{m})$, due to much stronger side erasing. At both edges, the output is considerably higher when the head is centered on the edge-microtrack for reading. This means that the MIG head is able to write signal more faraway from its gap edge than it can read. In other words, side writing is stronger than side reading. The edge-microtracks are very different for each side of the MIG head. The signal is decaying much faster at one edge causing an effective track 


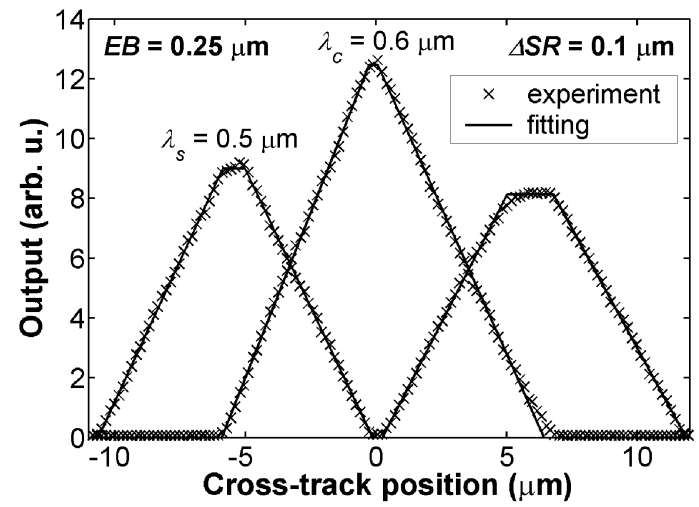

Fig. 6. Erase bands of HSS head measured from triple-track profiles.

width reduction around $1.5 \mu \mathrm{m}$ at a wavelength of $0.3 \mu \mathrm{m}$ (estimated using the center-microtracks as references). The effective track width reduction is considerably less at the other edge. It was found that the situation becomes the other way around when changing the tape moving direction. The side writing of the MIG head is anti-symmetric with respect to the tape moving direction. Each edge presents worse degradation in one direction than in the other, due to asymmetric write fields at the trailing edges. Side writing causes the transitions to bend and to broaden at the track edge. The bend of the transitions introduces a phase shift between the edge and the track-center, which contributes to the decrease of the signal at short wavelengths together with the transition broadening. The effects are strong at poor pole alignment and depend on the write field gradient at the trailing pole. These issues will be treated in more detail in a future publication.

The effective track width reduction induced by side writing effects causes the erase bands to broaden. It is therefore expected that the MIG head generates larger erase bands than the HSS head. The erase bands of the HSS and MIG heads were deduced from triple-track patterns using the interpretation technique described in [5]. A central track with wavelength $\lambda_{c}=0.6 \mu \mathrm{m}$ was used to overwrite with partial overlap two side tracks of wavelength $\lambda_{s}=0.5 \mu \mathrm{m}$. The experimental results are shown in Figs. 6 and 7 for each head. The HSS head exhibited erase bands as low as $0.25 \mu \mathrm{m}$, which are suitable for narrow track recording. The MIG head presented much larger erase bands of $0.76 \mu \mathrm{m}$, due to the poor alignment of its magnetic poles. Erase bands of the same order of magnitude were previously reported for MIG heads, extracted from MFM images [6]. The track profiles of the HSS head are better fitted by trapezoids than in the case of the MIG head, probably because its read response is closer to a square function. This is a consequence of the uniform gap and good pole alignment of the HSS

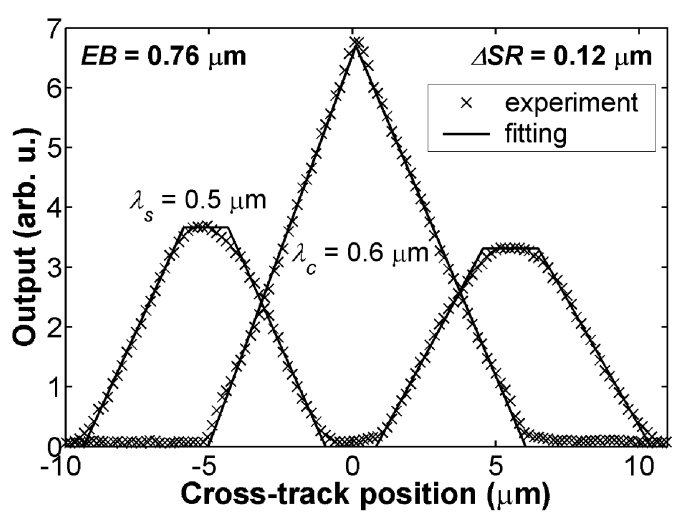

Fig. 7. Erase bands of MIG head measured from triple-track profiles.

head. The relative side read width, $\Delta S R$, is small and almost the same for both heads, as the wavelength of the central track is close to that of the side tracks.

\section{CONCLUSION}

A technique to investigate the side writing from the frequency response of microtracks was described. The method allows studying the degradation of the transitions at each track edge as a function of the frequency. It was applied to study the side writing of HSS and MIG heads, with identical electrical gap lengths of $0.23 \mu \mathrm{m}$. HSS heads presented very weak edge effects due to the good alignment of their magnetic poles. Their erase bands measured from triple-track profiles were around $0.25 \mu \mathrm{m}$ at $0.6 \mu \mathrm{m}$ wavelength, about three times smaller than those of the MIG heads. The erase bands could be further decreased by using shorter gap length, which is relatively easy to achieve in thin-film technology.

\section{REFERENCES}

[1] K. Tsuneki, K. Iesaka, Y. Yanagi, Y. Senshu, and Y. Kotani, "Development of a consumer VCR format and a high-density magnetic recording using an MR head," IEEE Trans. Consumer Electron., vol. 48, pp. 783-789, Aug. 2002.

[2] T. Ozue, M. Kondo, Y. Soda, S. Fukuda, S. Onodera, and T. Kawana, "11.5-Gb $/ \mathrm{in}^{2}$ recording using spin-valve heads in tape systems," IEEE Trans. Magn., vol. 38, pp. 136-140, Jan. 2002.

[3] L. T. Nguyen, A. Lisfi, and J. C. Lodder, "Obliquely sputtered $\mathrm{Co} / \mathrm{Cr}$ thin film tape for bidirectional recording," J. Appl. Phys., vol. 93, no. 10, pp. 7786-7788, May 2003.

[4] J. B. Albertini, H. Sibuet, P. Renaux, and P. Gaud, "A new solenoid magnetic integrated head for digital video recording," IEEE Trans. Magn., vol. 33, pp. 2836-2838, Sept. 1997.

[5] A. Hozoi, J. P. J. Groenland, J. B. Albertini, and J. C. Lodder, "Novel profiling model and side effects of helical scan silicon heads," IEEE Trans. Magn., vol. 38, pp. 1916-1918, Sept. 2002.

[6] N. Nagai, T. Shirai, S. Fukuda, T. Ozue, and S. Onodera, "A narrower side erase band for use with helical scan tape systems," IEEE Trans. Magn., vol. 35, pp. 2691-2693, Sept. 1999. 